\title{
Prediction of bleeding events using the VTE-BLEED risk score in patients with venous thromboembolism receiving anticoagulant therapy (Review)
}

\author{
MINERVA CODRUTA BADESCU ${ }^{1}$, MANUELA CIOCOIU ${ }^{2}$, OANA VIOLA BADULESCU ${ }^{2}$, \\ MARIA-CRISTINA VLADEANU ${ }^{2}$, IRIS BARARU BOJAN ${ }^{2}$, CRISTIANA ELENA VLAD $^{3}$ and CIPRIAN REZUS ${ }^{1}$ \\ Departments of ${ }^{1}$ Internal Medicine, ${ }^{2}$ Pathophysiology and ${ }^{3}$ Nephrology, \\ ‘Grigore T. Popa’ University of Medicine and Pharmacy, 700115 Iasi, Romania
}

Received July 2, 2021; Accepted August 3, 2021

DOI: 10.3892/etm.2021.10779

\begin{abstract}
Venous thromboembolism (VTE) is a major healthcare problem due to its high incidence, significant mortality rate from pulmonary embolism, high recurrence rate and morbidity from long-term complications. After a first episode of VTE all patients must receive anticoagulant treatment for 3 months. Further anticoagulation is recommended in patients without transient risk factors for VTE or patients with active cancer, if they are not at a high risk for bleeding. The VTE-BLEED risk score was created with the purpose of enabling a better stratification of the bleeding risk during stable anticoagulation after a first VTE. Currently, it is the most validated risk score in VTE settings (selected and non-selected cohorts). It has a good prediction power for major bleeding events in patients receiving any of the currently available classes of oral anticoagulants, and it can identify patients at risk of intracranial and fatal bleeding events. The aim of our review was to highlight the strengths of the VTE-BLEED risk score, to acknowledge its weak points and to properly position its use in current medical practice.
\end{abstract}

\section{Contents}

1. Introduction

2. Premises for a new bleeding risk score

3. Development and internal validation of the VTE-BLEED risk score

Correspondence to: Professor Manuela Ciocoiu or Dr Oana Viola Badulescu, Department of Pathophysiology, 'Grigore T. Popa' University of Medicine and Pharmacy, 16 Universitatii Street, 700115 Iasi, Romania

E-mail: mciocoiu2003@yahoo.com

E-mail: violabadulescu@yahoo.com

Key words: venous thromboembolism, pulmonary embolism, bleeding, risk score, VTE-BLEED, anticoagulants
4. External validation of the VTE-BLEED risk score

5. VTE-BLEED risk score performance in identifying patients at high risk of severe major bleedings

6. VTE-BLEED risk score performance in practice-based conditions

7. VTE-BLEED risk score performance in predicting in-hospital major bleedings

8. Conclusions

\section{Introduction}

Venous thromboembolism (VTE) encompasses deep vein thrombosis (DVT) and pulmonary embolism (PE) and is ranked third among the most common cardiovascular diseases, after acute coronary syndrome and stroke (1). It is recognized as a worldwide major healthcare problem due to its high incidence, significant mortality rate from PE $(2,3)$, high recurrence rate up to $30 \%$ at 10 years (4) and high morbidity through long-term complications (post-thrombotic syndrome and chronic thromboembolic pulmonary hypertension).

The main stream of treatment is anticoagulation. The current international guidelines recommend a minimal duration of 3 months of anticoagulant therapy after a first episode of VTE (5). Beyond this period, an individualized approach is sustained, weighing the risk of VTE recurrence once the treatment is discontinued to the risk of fatal or major bleeding during ongoing treatment. The risk of VTE recurrence after stopping anticoagulation is different after a provoked vs. an unprovoked thromboembolic event (6). Extended anticoagulation is currently recommended in patients without transient risk factors for VTE or patients with active cancer, if they are not at a high risk for bleeding (7). Therefore, the benefit-risk assessment of extended anticoagulation requires objective and reliable tools with which to predict both the VTE recurrence risk and the bleeding risk.

The fear of major bleeding is often present in the minds of the physicians prescribing anticoagulant treatment, but it should not be allowed to lead to unwarranted restraint from anticoagulant therapy. For this reason, various predictive models and hemorrhagic risk scores have been developed over 
time to aid in the therapeutic decision of anticoagulation. In 2016, a new score for bleeding risk assessment was proposed, the VTE-BLEED risk score, derived from a post hoc analysis of patients included in the RE-COVER (8) and RE-COVER II (9) trials. In the following years, the score underwent multiple external validations in selected and non-selected cohorts. With many promising results, the VTE-BLEED has all the chances to become the most widely used risk score in daily practice.

We conducted this review to highlight the strengths of the VTE-BLEED risk score, to acknowledge its weak points and to properly position its use in current medical practice. Automatic searches of Web of Science and Scopus databases were performed using the term 'VTE-BLEED' from January 2016 to October 2020. This key word was searched in the titles and abstracts of the articles. Review articles, meta-analyses and publications in a language other than English were excluded from further analysis. The reference lists of all remaining publications were assessed to retrieve relevant studies not identified by the initial search.

\section{Premises for a new bleeding risk score}

Major bleeding is the most relevant and frequent complication of therapeutic anticoagulation for VTE events, leading to increased morbidity and mortality. In patients enrolled in clinical trials, which are closely monitored, the bleedings are still a major concern and even more in unselected patients receiving usual care. The International Society of Thrombosis and Haemostasis (ISTH) defines major bleeding as fatal bleeding and/or symptomatic bleeding in a critical area or organ (intracranial, intraspinal, intraocular, retroperitoneal, intra-articular or pericardial, or intramuscular with compartment syndrome) and/or bleeding causing a fall in hemoglobin level of $\geq 2 \mathrm{~g} / \mathrm{dl}$ or leading to transfusion of $\geq 2$ units of whole blood or red cells (10).

Several clinical prediction scores and models for major bleeding are currently available. While some of them (HAS-BLED, HEMORR ${ }_{2}$ HAGES and ATRIA) were developed for patients with atrial fibrillation (11-13), others (RIETE, EINSTEIN, Hokusai, ACCP models) were specifically designed for VTE patients (14). Yet, no widely accepted prediction rule for VTE exists. VTE patients are usually younger than atrial fibrillation patients and have a different comorbidity profile (cancers are more prevalent than renal dysfunction), that could explain the limited usefulness of hemorrhagic risk scores derived from atrial fibrillation patients in the VTE settings. The RIETE score was designed to predict the risk of major bleeding only during the first 90 days of anticoagulation (15). The EINSTEIN model can be calculated only using a spreadsheet, thus being difficult to implement in current practice (16). The Hokusai score is validated only for edoxaban-using patients and the predictive power decreases after the first 3 months of treatment (17). The American College of Chest Physicians (ACCP) recommendations consist of a list of 18 variables identified from cohort studies (18), but incorporate poorly defined factors such as comorbidity and reduced functional capacity, and frequent falls.

Therefore, the VTE-BLEED risk score was developed at a time when there was no widely accepted and used bleeding risk scores for VTE patients, much less one directly focused on the hemorrhagic risk during extended anticoagulation. Based on the hypothesis that early bleeding events, included in previous risk assessment models, were not relevant for the prediction of bleeds during long-term and extended anticoagulant treatment, the VTE-BLEED score authors tried to identify those conditions that persist and influence the patient's hemorrhagic risk in the above mentioned time frame.

There are many data suggesting that the bleeding risk is not constant throughout the anticoagulant treatment period $(16,19)$. The highest occurrence of major bleeding is at the onset of anticoagulation and may reflect the importance of the patient's underlying diseases (20). Moreover, overlapping two types of anticoagulants in the very early phase of the treatment, either low-molecular-weight heparin (LMWH) or unfractionated heparin (UFH) with a vitamin $\mathrm{K}$ antagonist (VKA), raises the bleeding risk. In addition, due to the pharmacokinetic and pharmacodynamic properties of warfarin, the initial response to anticoagulant treatment may be variable and a stable anticoagulation may be achieved after up to several weeks $(18,21)$. Thus, for multiple reasons, some related to the patient's acute and chronic conditions and others related to the anticoagulation treatment itself, the bleeding risk is the highest in the first month after initiating anticoagulant treatment. Therefore, in developing this score, patients were considered on stable anticoagulation after the first 30 days of treatment following acute VTE, this new approach being rationale and fully justifiable.

\section{Development and internal validation of the VTE-BLEED risk score}

VTE-BLEED is a clinical risk score and it was developed based on a patient-level post hoc analysis of patients enrolled in two randomized controlled trials (RCTs), the RE-COVER (8) and RE-COVER II (9) trials. Only patients from dabigatran arms were included in the derivation analysis, due to the early stable effect of the drug in contrast to warfarin. All major and non-major clinically relevant bleeding events occurring during the 6 months of study were considered. Using logistic regression analysis, six variables were identified and included in the VTE-BLEED risk score, and each predictor received points according to the regression coefficients: active cancer (2p), male patients with uncontrolled arterial hypertension (1p), anemia (1.5p), history of bleeding ( $1.5 p)$, age $\geq 60$ years $(1.5 p)$ and renal dysfunction (1.5p) (22). The c-statistic for VTE-BLEED risk score was 0.72 (95\% CI, 0.67-0.76). The threshold was set at 2 points, a value that optimally differentiates patients with low ( $0-1.5$ points) and high ( $\geq 2$ points) bleeding risk. The risk of bleeding was 5 times higher in the high-risk category compared to the low-risk category (OR=5.0; 95\% CI, 3.5-7.1).

When applied to the warfarin group, the VTE-BLEED indicated a significantly lower statistical efficacy $(\mathrm{P}<0.001)$ compared to the dabigatran group, but still with a c-statistic above 0.5 (0.59; 95\% CI, 0.55-0.63) (22). When only major bleedings in patients on stable anticoagulation (after the first 30 days of treatment) were considered, good VTE-BLEED performance was noted, regardless of the anticoagulant type. Applying the 2 point threshold to dabigatran- and warfarin-treated patients, the risk of major bleeding was 6.5 times higher in the high-risk category compared to the 
low-risk category for both anticoagulants $(\mathrm{OR}=6.5 ; 95 \% \mathrm{CI}$, 2.0-21 for the dabigatran group and $\mathrm{OR}=6.5 ; 95 \% \mathrm{CI}, 2.8-15$ for the warfarin group) (22). The score also showed reliable performance when applied to dabigatran patients, regardless of whether they had acute PE or DVT, with a c-statistics of 0.74 (95\% CI, 0.67-0.80) and 0.71 (95\% CI, 0.65-0.76), respectively (22).

When VTE-BLEED, HAS-BLED, ATRIA and HEMORR ${ }_{2}$ HAGES scores were applied to the same dabigatran patient population (22) to assess their performance for predicting major bleeding events during stable anticoagulation, the VTE-BLEED score best identified patients at risk of bleeding (major and non-major clinically relevant bleeding) when considering the complete study period.

The advantages of the score quickly became visible. The VTE-BLEED score variables are clearly defined, easy to assess, largely objective and fairly constant over time (23). 'Male with uncontrolled arterial hypertension' is the only potentially inconstant variable. As a result, the score is more consistent and reliable than other bleeding risk scores that incorporates poorly defined or time-varying predictors $(18,24)$. Given that some variables can be found both in anticoagulant-related bleeding risk scores and VTE recurrence risk scores, it should be acknowledged that the VTE-BLEED score is free from this potential ambiguous effect as it does not include any predictor to be found in the VTE recurrence risk estimation scores $(25,26)$. Moreover, the binary risk stratification in high- and low-risk categories facilitates the therapeutic decisions regarding the extent of anticoagulation by excluding the ambiguity that usually exist in the management of patients from the 'intermediate risk' category.

\section{External validation of the VTE-BLEED risk score}

The accuracy of the VTE-BLEED risk score to predict major bleeding during stable anticoagulant treatment was investigated in patients from the Hokusai-VTE trial. This study enrolled patients with acute VTE and was designed to assess the efficacy and safety of edoxaban (60 mg once daily or $30 \mathrm{mg}$ once daily in selected patients) compared to standard treatment which consisted of LMWH or UFH followed by warfarin in dose adjusted according to International Normalized Ratio (INR).

Applying the 2 point threshold, the risk of major bleeding was constantly and significantly higher in the high-risk category compared to the low-risk category, with $\mathrm{OR}=5.04$ (95\% CI, 2.62-9.69) in the warfarin-treated patients group, with OR=3.09 (95\% CI, 1.54-6.22) in the edoxaban-treated patient group and with $\mathrm{OR}=4.04(95 \% \mathrm{CI}, 2.51-6.48)$ in the all study population (27). Patients with a total score of two points or more had a 4-fold increased risk of developing a major bleeding during stable anticoagulation compared to low-risk patients.

The score showed moderate predictive power for major bleeding in patients on stable anticoagulant treatment across all groups, with a c-statistic of 0.63 (95\% CI, 0.54-0.72) for the edoxaban group, 0.69 (95\% CI, 0.61-0.77) for the warfarin group and 0.66 (95\% CI, 0.61-0.72) for the all study population (27). Even if the score performed slightly poorer than in the derivation cohort, this should not be seen as a cause for concern or doubt. Validation of the risk score in its derivation cohort can be subjected to overestimation of the predictive accuracy (28). Moreover, there were relatively low number of major bleeding in both trials, and two of the determinants of the bleeding risk, active malignancy and history of bleeding, were more frequent in the RE-COVER trials than in Hokusai-VTE, with the possibility of influencing the final result.

Still, in all subgroup analyses (sex, age, patients with PE vs. DVT, with vs. without cancer, with vs. without chronic kidney disease, treatment length less than 180 days vs. 180 days or longer), the VTE-BLEED score was consistently able to identify patients at a 2.5 - to 11 -fold higher risk of bleeding.

This validation study is important at least for three reasons. First, the performance of the score was assessed in patients who received anticoagulant treatment for more than 6 months, due to the 12-month follow-up period of the Hokusai VTE trial. Second, the direct anticoagulant used in Hokusai-VTE was edoxaban, a factor Xa inhibitor, which allowed the evaluation of the score performance in a population anticoagulated differently than RE-COVER trial patients that received dabigatran, a factor IIa inhibitor. Third, subgroup analyses demonstrated the good predictive power of the score across all relevant patient subcategories.

As in the derivation cohort, the Hokusay VTE trial excluded patients with creatinine clearance $<30 \mathrm{ml} / \mathrm{min}$; therefore, data regarding the performance of the score in this category of patients were lacking.

\section{VTE-BLEED risk score performance in identifying patients at high risk of severe major bleedings}

The most severe hemorrhagic events, intracranial (ICH) and fatal bleedings, raise the highest concerns (29). Therefore, the performance of the VTE-BLEED risk score to identify patients at risk of developing these complications during stable oral anticoagulation after a first episode of VTE was assessed in patients enrolled in the RE-COVER and Hokusai-VTE trials. In this large population, three outcomes were analyzed: $\mathrm{ICH}$, fatal bleeding and a composite outcome of $\mathrm{ICH}$ or fatal bleeding. Although the incidence of those events was low in all studies $(8,9,30)$, the VTE-BLEED score showed a good prediction ability. The ORs for ICH were 4.4 (95\% CI, 0.74-26), 3.8 (95\% CI, 1.5-10) and 4.0 (95\% CI, 1.7-9.3) in RE-COVER, Hokusai-VTE and all study population, respectively (31). The OR for fatal bleeding were 4.4 (95\% CI, 0.74-26), 6.7 (95\% CI, 1.3-35) and 5.6 (95\% CI, 1.7-19) in the RE-COVER, Hokusai-VTE and all study population, respectively (31). Finally, the ORs for ICH or fatal bleeding were 4.9 (95\% CI, 1.2-21), 4.6 (95\% CI, 1.8-12) and 4.7 (95\% CI, 2.2-10) in RE-COVER, Hokusai-VTE and all study population, respectively (31). The OR for all three outcomes ranged between 3.8 and 6.7 in the RE-COVER and Hokusai-VTE trials, and between 4.0 and 5.6 in polled data, supporting the value of the score in identifying patients at risk for the most severe major bleeding events (ICH and fatal bleeding), which is more important than predicting major bleeding in general. Bleeding risk assessment based on hard clinical outcome ( $\mathrm{ICH}$ and fatal bleeding) is crucial in patients with a high risk for recurrent VTE in whom tailoring the duration of anticoagulant treatment assumes continuing the 
anticoagulant therapy as long as the benefit in the prevention of recurrent thrombosis outweighs the risk of bleeding events.

\section{VTE-BLEED risk score performance in practice-based conditions}

Patients enrolled in RCTs are highly selected due to the rigorous inclusion criteria; therefore, they usually have less severe comorbidities and a lower risk of hemorrhage compared to patients with VTE encountered in current practice. To overcome this aspect, evaluation of the score performance in 'real-world' cohorts is of high importance.

The VTE-BLEED risk score ability to identify patients with high bleeding risk under stable anticoagulation was assessed in patients from the prospective XALIA study. Patients with DVT alone or with concomitant acute PE received either rivaroxaban or conventional anticoagulant therapy (parenteral anticoagulants followed by a VKA) and were followed up for at least 12 months. In this population, the VTE-BLEED risk score was able to differentiate patients with high-from those with low-risk of major bleeding during stable anticoagulation, with an OR for 1-point score increase of 1.4 (95\% CI, 1.2-1.7) in the overall studied population, and without significant differences related to sex, age or the type of anticoagulant used (32). The ORs for 1-point score increase were 1.5 (95\% CI, 1.1-2.2) and 1.3 (95\% CI, 1.1-1.6) in the rivaroxaban and VKA arm, respectively (32). Particular for cancer patients, increases in the score were less predictive for major bleeding, with an OR of 1 (95\% CI, 0.61-1.7) most likely because they were all from the beginning in a 'high-risk' category, as the diagnosis of active cancer is scored with 2 points. When only major bleeding events occurring after 90 days of anticoagulation were considered, the c-statistic improved from 0.68 to 0.71 (32). The OR for 1-point score remained 1.4 (95\% CI, 1.1-1.7) in the overall studied population. The c-statistic also improved from 0.64 to 0.71 in patients with unprovoked VTE, a category of patients for whom accurate prediction of major bleeding on long-term anticoagulation was most relevant. As the score contains 'active cancer' as a major predictor, the performance of the score could have been lower in patients with unprovoked VTE, but the results proved the opposite and strengthened the value of the score.

In a non-selected cohort of consecutive patients with acute symptomatic VTE from the COMMAND VTE (COntemporary ManageMent AND outcomes in patients with Venous ThromboEmbolism) Registry, a large observational real-world Japanese database, the cumulative 5-year incidence of major bleeding in patients from the high-vs. low-risk groups of VTE-BLEED risk score was compared. The cumulative 5-year incidence of major bleeding during stable anticoagulation with VKA or DOAC (apixaban, dabigatran, edoxaban, rivaroxaban) was significantly statistically higher in the high-risk group than in the low-risk group (13.2 vs. 5.4\%, $\mathrm{P}<0.001)$ (33). The VTE-BLEED risk score successfully identified patients with high-risk of major bleeding during chronic anticoagulant treatment and also those with high-risk of ICH or fatal bleeding. The cumulative 5-year incidence of ICH or fatal bleeding were $4.1 \%$ in high-risk patients and $1.4 \%$ in low-risk patients $(\mathrm{P}=0.073)$ (33).

In an Italian prospective cohort of consecutive patients with VTE receiving DOAC, the VTE-BLEED risk score accurately identified the patients with increased bleeding risk. The patients from the high-risk category had a significantly higher risk of major bleedings compared to the patients from the low-risk category (HR 16.11; 95\% CI, 2.18-119.09; $\mathrm{P}=0.006$ ). It should be noted that $15.9 \%$ of patients had active cancer and $14.4 \%$ had creatinine clearance $<50 \mathrm{ml} / \mathrm{min}$. Despite the wide confidence interval and the modest c-statistics of 0.674 (95\% CI, 0.593-0.755) for the entire 12-month study period, the score still has an acceptable discrimination power (34). The highest differences in the occurrence of major bleeding between high- and low-risk categories were recorded in patients with DVT only (3.6 vs. $0.5 \%$; HR 8.71; 95\% CI, 1.12-67.45) and in those with unprovoked VTE (3.6 vs. $0.4 \%$; HR 9.86; $95 \%$ CI, 1.28-75.80). Similarly to previous results (23), no significant increase in bleeding events was observed in cancer patients compared to those without cancer (HR 1.93; 95\% CI, 0.72-5.17; $\mathrm{P}=0.191$ ).

The VTE-BLEED risk score ability to predict the bleeding risk in high-compared to low-risk category was recently assessed in patients with VTE from the RIETE Registry (35). The low discriminative power of the score (c-statistic of 0.56 ) could be the result of the small number of patients with major bleeding events in the studied population ( 8 patients in the low-risk category and 6 patients in the high-risk category). In support of this hypothesis is the equally low performance of the RIETE and HAS-BLED risk scores when applied to the same population.

VKA and DOAC are different in terms of pharmacokinetic and pharmacodynamic properties. This fact influences their safety profile (the general hemorrhagic risk and the prevalent bleeding site) and could also change the importance of some predictors within the bleeding risk scores. For instance, labile INR has impact only on VKA-treated patients (36), while in patients receiving DOAC the status of renal function is of great importance (37). Therefore, some predictors could weigh more and significantly influence the bleeding risk. Nevertheless the VTE-BLEED risk score showed good ability to differentiate patients with high-vs. low-risk of bleeding irrespective of oral anticoagulant type, VKA or DOAC.

In patients with a VTE-BLEED risk score $\geq 2$ points, the duration of anticoagulant treatment is expected to be shorter compared to patients at low-risk. If patients at high risk of bleeding would have an increased rate of recurrent thromboembolic events due to withdrawal of anticoagulation, the utility of the VTE-BLEED risk score would be questionable. This aspect has a vital importance in patients requiring indefinite anticoagulation and having a high-risk of bleeding. For this reason, the incidence of recurrent VTE after discontinuation of anticoagulation was assessed post hoc in the PADIS-PE study population (23). Patients with a first unprovoked PE were enrolled and received 6 or 24 months of VKA treatment. Active cancer was an exclusion criterion of this trial. During a 24-month follow-up period after cessation of anticoagulation, the cumulative incidence of recurrent VTE was 16\% (95\% CI, 10.0-26.1) in the high-risk VTE-BLEED group and 15\% (95\% CI, 10.4-20.3) in the low-risk VTE-BLEED group (23). This finding indicated that patients with a VTE-BLEED risk score $\geq 2$ points did not have a higher risk of developing recurrent VTE than patients with a VTE-BLEED score <2 points (adjusted HR 1.16; 95\% CI, 
0.62-2.19), that supports the utility of this score in appreciating the duration of anticoagulant treatment according to the individual bleeding risk in patients without cancer. Further evidence is needed for cancer patients.

\section{VTE-BLEED risk score performance in predicting in-hospital major bleedings}

Although designed and validated to identify patients with high hemorrhagic risk during stable oral anticoagulation, the VTE-BLEED risk score performed better than HAS-BLED in identifying patients with a high risk of major bleeding during in-hospital anticoagulation for a first acute PE. Both scores were applied on patients from the real-world cohort of PERGO (Pulmonary Embolism Registry of Goettingen). While HAS-BLED risk score $\geq 2$ points was not able to predict major bleeding (OR, 1.1; 95\% CI, 0.4-2.9), a VTE-BLEED risk score of $\geq 2$ points was a good predictor of in-hospital major bleeding events with OR 3.7 (95\% CI, 1.1-13.0) (20).

The ability of four bleeding risk scores (HAS-BLED, HEMORR ${ }_{2}$ HAGES, RIETE and VTE-BLEED) to predict major and clinically relevant non-major bleeding events during in-hospital anticoagulation for acute VTE were assessed in the PE-aWARE registry, a real-world cohort of unselected patients. The VTE-BLEED, RIETE and HEMORR ${ }_{2} \mathrm{HAGES}$ risk scores had similar predictive power, with a c-statistic of 0.75 (95\% CI, 0.70-0.80), 0.77 (95\% CI, 0.72-0.81) and 0.76 (95\% CI, 0.70-0.80), respectively. All performed better than the HAS-BLED risk score, which had a c-statistic of 0.51 (95\% CI, 0.46-0.57) (38). The different performance was probably due to the fact that the variable 'cancer' is not part of the HAS-BLED risk score as in the other three scores. Combining the score with the values of D-Dimer measured at admission, as an expression of clot burden and severity of the coagulation system alterations, the prediction ability of RIETE, HEMORR ${ }_{2}$ HAGES and HAS-BLED risk scores was improved, but not that of the VTE-BLEED risk score, probably due to the fact that the VTE-BLEED risk score was designed and validated for assessing bleeding risk beyond 30 days from the acute VTE.

\section{Conclusions}

Treatment duration needs to be appreciated on an individual basis in patients requiring extended anticoagulation. Therefore identifying high-risk bleeding patients is mandatory and demands reliable tools. The VTE-BLEED risk score was created with the purpose of enabling a better stratification of the bleeding risk during stable anticoagulation after a first VTE.

It uses six simple and objective clinical variables, easy to assess. None of its predictors are part of the VTE recurrence risk estimation scores; therefore, the VTE-BLEED risk score is free from this potential ambiguous effect. The binary risk stratification in high- and low-risk categories excludes the ambiguity surrounding the management of patients from the 'intermediate risk' category, thus helping the therapeutic decision.

It is the most validated bleeding risk score in VTE patients. It was derived and initially validated in the setting of RCTs, and then it was externally validated in another RCT, in a selected cohort and in non-selective cohorts. It has the ability to identify patients at high risk of major bleeding during stable anticoagulant treatment, regardless if they have PE or DVT, and for any of the currently available classes of oral anticoagulants. The score can reliable identify patients at risk of ICH and fatal bleeding events, which is more important than just predicting major bleeding in general.

The score limitation should also be acknowledged. The score has not been studied in patients with recurrent thromboembolism, which is why the performance of the score in this category of patients is not known. Although the score contains the 'active cancer' parameter, there is no stratification of the risk according to the type of neoplasia in order to increase the accuracy of the score in the neoplastic population.

Although there is still a lot to be known in determining the risk of major bleeding during long-term and extended anticoagulant therapy, the VTE-BLEED risk score has accumulated over time an extensive body of evidence to support its clinical utility.

\section{Acknowledgements}

Not applicable.

\section{Funding}

No funding was received.

\section{Availability of data and materials}

All information provided in this article is documented by relevant references.

\section{Authors' contributions}

All authors substantially contributed to this review paper. Conception and design were the responsibility of MCB, MC and CR. Acquisition of data was carried out by OVB, MCV, $\mathrm{CV}$, and IBB. Analysis and interpretation of the data were the responsibility of $\mathrm{MCB}, \mathrm{OVB}, \mathrm{MCV}, \mathrm{CV}$, and $\mathrm{IBB}$. Drafting the manuscript and revising it critically for important intellectual content were carried out by MCB, MC, and CR. All authors read and approved the final manuscript for publication.

\section{Ethics approval and consent to participate}

Not applicable.

\section{Patient consent for publication}

Not applicable.

\section{Competing interests}

The authors declare that they have no competing interests.

\section{References}

1. Gillum RF: Pulmonary embolism and thrombophlebitis in the United States, 1970-1985. Am Heart J 114: 1262-1264, 1987. 
2. Heit JA, Silverstein MD, Mohr DN, Petterson TM, Lohse CM, O'Fallon WM and Melton LJ III: The epidemiology of venous thromboembolism in the community. Thromb Haemost 86: 452-463, 2001

3. Heit JA: The epidemiology of venous thromboembolism in the community: Implications for prevention and management. J Thromb Thrombolys 21: 23-29, 2006.

4. Heit JA: Predicting the risk of venous thromboembolism recurrence. Am J Hematol 87 (Suppl 1): S63-S67, 2012.

5. Kearon C, Akl EA, Ornelas J, Blaivas A, Jimenez D, Bounameaux H, Huisman M, King CS, Morris TA, Sood N, et al: Antithrombotic therapy for VTE disease: CHEST guideline and expert panel report. Chest 149: 315-352, 2016.

6. Kearon C, Ageno W, Cannegieter SC, Cosmi B, Geersing GJ and Kyrle PA: Categorization of patients as having provoked or unprovoked venous thromboembolism: Guidance from the SSC of ISTH. J Thromb Haemost 14: 1480-1483, 2016.

7. Konstantinides SV, Meyer G, Becattini C, Bueno H, Geersing GJ, Harjola VP, Huisman MV, Humbert M, Jennings CS Jiménez D, et al: 2019 ESC Guidelines for the diagnosis and management of acute pulmonary embolism developed in collaboration with the European Respiratory Society (ERS). Eur Heart J 41: 543-603, 2020

8. Schulman S, Kearon C, Kakkar AK, Mismetti P, Schellong S, Eriksson H, Baanstra D, Schnee J and Goldhaber SZ; RE-COVER Study Group: Dabigatran versus warfarin in the treatment of acute venous thromboembolism. N Engl J Med 361: 2342-2352, 2009.

9. Schulman S, Kakkar AK, Goldhaber SZ, Schellong S, Eriksson H, Mismetti P, Christiansen AV, Friedman J, Le Maulf F, Peter N, et al: Treatment of acute venous thromboembolism with dabigatran or warfarin and pooled analysis. Circulation 129: 764-772, 2014

10. Schulman S and Kearon C: Definition of major bleeding in clinical investigations of antihemostatic medicinal products in non-surgical patients. J Thromb Haemost 3: 692-694, 2005

11. Pisters R, Lane DA, Nieuwlaat R, de Vos CB, Crijns HJ and Lip GY: A novel user-friendly score (HAS-BLED) to assess 1-year risk of major bleeding in patients with atrial fibrillation: The Euro Heart Survey. Chest 138: 1032-1033, 2010.

12. Gage BF, Yan Y, Milligan PE, Waterman AD, Culverhouse R, Rich MW and Radford MJ: Clinical classification schemes for predicting hemorrhage: Results from the National Registry of Atrial Fibrillation (NRAF). Am Heart J 151: 713-719, 2006.

13. Singer DE, Chang Y, Borowsky LH, Fang MC, Pomernacki NK, Udaltsova N, Reynolds K and Go AS: A new risk scheme to predict ischemic stroke and other thromboembolism in atrial fibrillation: The ATRIA study stroke risk score. J Am Heart Assoc 2: e000250, 2013.

14. Klok FA, Kooiman J, Huisman MV, Konstantinides S and Lankeit M: Predicting anticoagulant-related bleeding in patient with venous thromboembolism: A clinically oriented review. Eur Respir J 45: 201-210, 2015.

15. Ruíz-Giménez N, Suárez C, González R, Nieto JA, Todolí JA, Samperiz AL and Monreal M; RIETE Investigators: Predictive variables for major bleeding events in patients presenting with documented acute venous thromboembolism. Findings from the RIETE Registry. Thromb Haemost 100: 26-31, 2008.

16. Di Nisio M, Ageno W, Rutjes AW, Pap AF and Büller HR: Risk of major bleeding in patients with venous thromboembolism treated with rivaroxaban or with heparin and vitamin $\mathrm{K}$ antagonists. Thromb Haemost 115: 424-432, 2016.

17. Di Nisio M, Raskob G, Büller HR, Grosso MA, Zhang G, Winters SM and Cohen A: Prediction of major and clinically relevant bleeding in patients with VTE treated with edoxaban or vitamin K antagonists. Thromb Haemost 117: 784-793, 2017.

18. Ageno W, Gallus AS, Wittkowsky A, Crowther M, Hylek EM and Palareti G: Oral anticoagulant therapy: Antithrombotic therapy and prevention of thrombosis, 9th ed: American College of Chest Physicians Evidence-Based Clinical Practice Guidelines. Chest 141 (Suppl 2): e44S-e88S, 2012

19. Klok FA, Niemann C, Dellas C, Hasenfuß G, Konstantinides S and Lankeit M: Performance of five different bleeding-prediction scores in patients with acute pulmonary embolism. J Thromb Thrombolysis 41: 312-320, 2016

20. Kresoja KP, Ebner M, Rogge NIJ, Sentler C, Keller K Hobohm L, Hasenfuß G, Konstantinides SV, Pieske B and Lankeit M: Prediction and prognostic importance of in-hospital major bleeding in a real-world cohort of patients with pulmonary embolism. Int J Cardiol 290: 144-149, 2019.

21. Kangelaris KN, Bent S, Nussbaum RL, Garcia DA and Tice JA: Genetic testing before anticoagulation? A systematic review of pharmacogenetic dosing of warfarin. J Gen Intern Med 24 $656-664,2009$
22. Klok FA, Hösel V, Clemens A, Yollo WD, Tilke C, Schulman S, Lankeit $M$ and Konstantinides SV: Prediction of bleeding events in patients with venous thromboembolism on stable anticoagulation treatment. Eur Respir J 48: 1369-1376, 2016.

23. Klok FA, Presles E, Tromeur C, Barco S, Konstantinides SV, Sanchez O, Pernod G, Raj L, Robin P, Le Roux PY, et al: Evaluation of the predictive value of the bleeding prediction score VTE-BLEED for recurrent venous thromboembolism. Res Pract Thromb Haemost 3: 364-371, 2019.

24. Brown JD, Goodin AJ, Lip GYH and Adams VR: Risk stratification for bleeding complications in patients with venous thromboembolism: Application of the HAS-BLED bleeding score during the first 6 months of anticoagulant treatment. J Am Heart Assoc 7: e007901, 2018.

25. Eichinger S, Heinze G, Jandeck LM and Kyrle PA: Risk assessment of recurrence in patients with unprovoked deep vein thrombosis or pulmonary embolism: The Vienna prediction model. Circulation 121: 1630-1636, 2010.

26. Tosetto A, Iorio A, Marcucci M, Baglin T, Cushman M, Eichinger S, Palareti G, Poli D, Tait RC and Douketis J: Predicting disease recurrence in patients with previous unprovoked venous thromboembolism: A proposed prediction score (DASH). J Thromb Haemost 10: 1019-1025, 2012

27. Klok FA, Barco S and Konstantinides SV: External validation of the VTE-BLEED score for predicting major bleeding in stable anticoagulated patients with venous thromboembolism. Thromb Haemost 117: 1164-1170, 2017.

28. Shipe ME, Deppen SA, Farjah F and Grogan EL: Developing prediction models for clinical use using logistic regression: An overview. J Thorac Dis 11 (Suppl 4): S574-S584, 2019.

29. Morotti A and Goldstein JN: Anticoagulant-associated intracerebral hemorrhage. Brain Hemorrhages 1: 89-94, 2020.

30. Hokusai-VTE Investigators; Büller HR, Décousus H, Grosso MA, Mercuri M, Middeldorp S, Prins MH, Raskob GE, Schellong SM, Schwocho L, et al: Edoxaban versus warfarin for the treatment of symptomatic venous thromboembolism. N Engl J Med 369: 1406-1415, 2013

31. Klok FA, Barco S and Konstantinides SV: Evaluation of VTE-BLEED for predicting intracranial or fatal bleeding in stable anticoagulated patients with venous thromboembolism. Eur Respir J 51: 1800077, 2018.

32. Klok FA, Barco S, Turpie AGG, Haas S, Kreutz R, Mantovani LG, Gebel M, Herpers M, Bugge JP, Kostantinides SV and Ageno W: Predictive value of venous thromboembolism (VTE)-BLEED to predict major bleeding and other adverse events in a practice-based cohort of patients with VTE: Results of the XALIA study. Br J Haematol 183: 457-465, 2018.

33. Nishimoto Y, Yamashita Y, Morimoto T, Saga S, Amano H, Takase T, Hiramori S, Kim K, Oi M, Akao M, et al: Validation of the VTE-BLEED score's long-term performance for major bleeding in patients with venous thromboembolisms: From the COMMAND VTE registry. J Thromb Haemost 18: 624-632, 2020

34. Vedovati MC, Mancuso A, Pierpaoli L, Paliani U, Conti S, Ascani A, Galeotti G, Di Filippo F, Caponi C, Agnelli G and Becattini C: Prediction of major bleeding in patients receiving DOACs for venous thromboembolism: A prospective cohort study. Int J Cardiol 301: 167-172, 2020.

35. Hidalgo Soto M, Zamanillo Herreros I, Diaz Pedroche C, Gómez Cuervo C, Vera Guerrero E, Lóopez Muñoz N, De Nicolás Sol R, Gutierrez de Ocariz X, Buendía Ureña B, Iñiguez García R, et al: VTE-BLEED score to predict bleeding complications in patients with venous thromboembolism: From the RIETE Registry. Virtual Edition of the 25th European Hematology Association (EHA) Annual Congress: S324, 2020.

36. Proietti M, Senoo K, Lane DA and Lip GY: Major bleeding in patients with non-valvular atrial fibrillation: Impact of time in therapeutic range on contemporary bleeding risk scores. Sci Rep 6: 24376, 2016.

37. Becattini C, Giustozzi M, Ranalli MG, Bogliari G, Cianella F, Verso M, Agnelli G and Vedovati MC: Variation of renal function over time is associated with major bleeding in patients treated with direct oral anticoagulants for atrial fibrillation. J Thromb Haemost 16: 833-841, 2018

38. Skowrońska M, Furdyna A, Ciurzyński M, Pacho S, Bienias P, Palczewski P, Kurnicka K, Jankowski K, Lipińska A, Uchacz K, et al: D-dimer levels enhance the discriminatory capacity of bleeding risk scores for predicting in-hospital bleeding events in acute pulmonary embolism. Eur J Int Med 69: 8-13, 2019. 